\title{
A single-season prospective study of respiratory viral infections in lung transplant recipients
}

\author{
A.P. Milstone*,", L.M. Brumble*, J. Barnes`, W. Estes ${ }^{+}$, J.E. Loyd**\#, \\ R.N. Pierson III ${ }^{\uparrow, \#}$ and S. Dummer, ${ }^{*}, \oplus_{, \#}$
}

ABSTRACT: The frequency and complications of respiratory viral infections (RVI) were studied in 50 ambulatory lung transplant patients during a single winter season, using viral antigens, viral cultures and PCR of nasal washes or bronchoalveolar lavages. Patients' survival, episodes of acute rejection and occurrence of bronchiolitis obliterans (BO) or BO syndrome (BOS) were monitored for $1 \mathrm{yr}$ after the study.

Overall, 32 (64\%) patients had 49 symptomatic episodes. Documented infections included eight due to respiratory syncytial virus (RSV), one due to parainfluenza virus (PIV) and 10 due to influenza (FLU). Four of the FLU infections were serological rises without symptoms. Overall, 17 (34\%) patients had documented viral infection; four patients had lower respiratory involvement and two (one RSV, one PIV) were hospitalised for aerosolised ribavirin treatment.

After $1 \mathrm{yr}$ there were three (6\%) deaths unrelated to RVI. BO or BOS had occurred in one (6\%) out of 17 patients with and three (12\%) out of 33 without RVI. Respiratory viruses infected onethird of ambulatory lung transplant recipients in a single season.

In conclusion, respiratory viral infection was not associated with subsequent graft dysfunction. Larger prospective studies are required to better define the acute and long-term morbidity of these infections.

KEYWORDS: Lung transplantation, PCR, respiratory virus

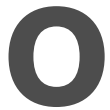
nly a few infections caused by respiratory viruses were reported in the initial studies of infectious complications of lung transplantation $[1,2]$. Subsequently, studies that focused on respiratory viral infection documented cumulative infection rates ranging $8-21 \%$ in populations of lung transplant recipients followed over 5-7-yr intervals [3-5]. The average yearly incidence of respiratory viral infection in these studies was $2-3 \%$. The mortality rate in two studies in which this was reported was $10 \%[3,4]$. There appear to be differences in severity and outcome related to the type of viral infection. For instance, rhinovirus infections were not commonly associated with symptoms, but adenovirus infections were associated with a high rate of respiratory failure and death [5-8].

Apart from the direct morbidity and mortality caused by respiratory viral infections, there has been a concern that respiratory viral infections might also cause chronic allograft dysfunction due to bronchiolitis obliterans (BO) in lung allografts by stimulating alloimmune cells in the lung. This possibility is supported by an animal model [9]. There is also clinical evidence supporting such a relationship from retrospective studies $[3,10,11]$. In these studies, the diagnoses were made by viral culture of bronchoalveolar lavage fluid (BALF) in patients who underwent bronchoscopy for lower respiratory tract symptoms or a documented change in pulmonary function. Such a retrospective approach is likely to detect the most severe cases, but may underestimate the actual rate of infection. Also, patients with pre-existing lung dysfunction may be more likely to be detected in such a study. Moreover, available studies have usually employed only one or two diagnostic methods to detect infection, such as culture or viral antigen testing. Additional diagnostic tools such as serology and PCR that have been shown to increase the rate of diagnosis of respiratory pathogens in some population studies have not routinely been used [12-15].

To better understand the epidemiology of respiratory viral infections in a lung transplant
AFFILIATIONS

Depts of *Medicine and

"Surgery, and

\#Vanderbilt Transplant Center, and

${ }^{+}$Clinical Virology Laboratory,

Vanderbilt University Hospital,

Vanderbilt University School of

Medicine, Nashville, TN, USA.

CORRESPONDENCE

S. Dummer

911 Oxford House

Vanderbilt University

Nashville

TN 37232

USA

Fax: 16159361970

E-mail: stephen.dummer@

vanderbilt.edu

Received:

September 082005

Accepted after revision:

February 212006

\section{SUPPORT STATEMENT}

The present study received a funding grant from V. Riddle and

Medlmmune, Inc. (Gaithersburg, MD, USA) 
population, the current authors undertook an intensive, multiple-modality, prospective study of respiratory viral infections in lung recipients in a single winter viral season in Nashville (TN, USA). The aims of the study were to: 1) obtain an accurate rate of infection of the most important respiratory viruses in lung recipients; 2) document the short-term morbidity and mortality of these infections; and 3) determine the incidence of acute rejection, new diagnoses of $\mathrm{BO}$ and death in both infected and uninfected patients during a 1-yr follow-up.

\section{METHODS}

\section{Clinical}

From November 1, 1999 until March 31, 2000, lung transplant recipients at Vanderbilt University Medical Center (Nashville) were recruited for a prospective evaluation of viral respiratory infection. Adult patients were eligible for enrolment if they were $\geqslant 2$ weeks post-transplant, being followed in the outpatient clinic, and lived within $2 \mathrm{~h}$ driving time of the medical centre. The study was closed after 50 patients were enrolled. No participant in the study received oral antiviral prophylaxis for influenza (FLU; i.e. amantadine or ramantadine). The study was supported by a research grant from MedImmune, Inc. (Gaithersburg, MD, USA). All patients who participated in the study gave informed consent and signed consent forms approved by the Vanderbilt University Medical Center Institutional Review Board. A study nurse interviewed each patient weekly by telephone or in clinic to screen for signs and symptoms of a new respiratory infection. Symptoms monitored included both upper respiratory tract symptoms (e.g. coryza, stuffy nose, sore throat and sneezing) and lower respiratory tract symptoms (e.g. cough, wheezing, shortness of breath). Patients with active symptoms were seen within $48 \mathrm{~h}$ in the clinic where a physical examination and a nasal wash (NW) were performed. For the NW, $5 \mathrm{~mL}$ of nonbacteriostatic saline was instilled into each nostril and collected in a sterile cup. An aliquot of this sample was submitted to the Vanderbilt University Hospital virology laboratory for viral culture and viral antigen testing. The remainder of the NW was frozen at $-70^{\circ} \mathrm{C}$ for future PCR testing. In addition, all patients had serum drawn at enrolment and between March 28, 2000, and July 7, 2000, when they visited the clinic for routine follow-up (mean 115.8 \pm 35.4 days). Patients who underwent bronchoscopy and bronchoalveolar lavage (BAL) also had BALF submitted for viral culture, viral antigens and an aliquot frozen for PCR testing. A few patients were admitted directly to hospital and underwent bronchoscopy. These individuals did not have NW and only BALF was available for analysis. The decision to perform bronchoscopy and the treatment of the patient's illness was determined by the attending pulmonary transplant service physician. Patients were not routinely studied for cytomegalovirus infection unless they were $<6$ months from transplant, in which case they were managed by the current authors' standard cytomegalovirus regimen, which relies on a brief 2-week period of antiviral prophylaxis followed by monitoring of blood antigenemia and pre-emptive therapy [16].

The primary outcome was the occurrence of any respiratory tract viral infection. Secondary outcomes included the occurrence of viral lower respiratory tract infection (LRTI) or pneumonia, as well as the need for hospitalisation and death within 30 days. Patients were evaluated for 1 yr after the end of the study for the occurrence of acute rejection, new diagnoses of BO or BO syndrome (BOS), or death. The diagnosis of LRTI required detection of a virus and the presence of new signs suggesting lower respiratory tract involvement (e.g. wheezing, rales or rhonchi over the transplant lung), together with shortness of breath, new hypoxaemia or $>10 \%$ decrease in forced expiratory volume in one second. The diagnosis of pneumonia additionally required the appearance of a new infiltrate on chest radiograph or computerised tomographical scan and a transbronchial biopsy showing evidence for parenchymal infection. Acute rejection was diagnosed by transbronchial biopsy and BO and BOS were diagnosed using the criteria established by the International Society of Heart and Lung Transplantation [17].

As the present prospective study was only performed during a single respiratory viral season the current authors wanted to assess whether the rate of viral infection in the community during that year differed from other years. Therefore, data was collected on the frequency and proportion of positive FLU cultures and respiratory syncytial virus (RSV) antigen tests for five consecutive respiratory viral seasons from the Vanderbilt University Hospital virology laboratory (Nashville). The hospital virology laboratory performs all virological testing from adults and children at Vanderbilt University Medical Center and also receives many samples from local hospitals and clinics. The data included test results for 5-month intervals (November 1999 to March 2000) for 2 yrs prior to and 2 yrs after the 1999-2000 viral season. Culture data for RSV could not be analysed because in August 1999 the virology lab stopped performing RSV cultures when RSV antigens were positive on samples requesting RSV antigen only.

\section{Laboratory}

NW and BALF were inoculated into culture tubes of Hep-2 and rhesus monkey kidney (RMK) cells (BioWhitaker Inc., Walkersville, MD, USA) and examined for cytopathological effect (CPE). CPE in Hep-2 cells was stained with Chemicon anti-RSV followed by fluorescein isothiocyanate (FITC)-conjugated goat anti-mouse immunoglobulin (Ig)G. RMK cells were additionally assayed for haemadsorption of guinea pig red blood cells at days 5 and 10. If CPE or haemadsorption was detected, the cells were stained with Chemicon antibodies against FLU A and B and parainfluenza virus (PIV) 1, 2 and 3, followed by FITC-conjugated goat anti-mouse IgG. Samples were additionally tested by RSV Directigen and FLU A and B Directigen (Becton Dickinson, Franklin Lakes, NJ, USA). As performed, the viral cultures would be expected to detect adenoviruses, but not rhinoviruses. PCR for RSV A and B, FLU $A$ and $B$, and PIV 1, 2 and 3 were performed by hexaplex PCR (Prodesse, Milwaukee, WI, USA), as previously described [13]. Pre- and post-study sera were assayed for RSV antibodies by ELISA by a previously described assay; however, purified RSV F protein was substituted for G protein [18]. FLU antibodies to A/Beijing/262/95, A/Sydney/5/97 and B/Beijing/184/93 were measured by kinetic ELISA using a modification of a previously described assay [19]. An increase of the kinetic ELISA of $\geqslant 100 \mathrm{mOD} \cdot \mathrm{min}^{-1}$ was used as a criterion of seroconversion. In order to exclude an effect from the FLU 


\section{TABLE 1 Patient demographics}

\begin{tabular}{|c|c|c|}
\hline Characteristic & Infected & Uninfected \\
\hline Subjects & 17 & 33 \\
\hline Age yrs & $50 \pm 13$ & $50 \pm 10$ \\
\hline Sex M:F & $12: 5$ & $16: 17$ \\
\hline \multicolumn{3}{|l|}{ Pre-transplant diagnosis } \\
\hline IPF & $7(41)$ & $9(27)$ \\
\hline COPD & $4(24)$ & $10(30)$ \\
\hline AAT & $1(8)$ & $3(9)$ \\
\hline $\mathrm{CF}$ & $2(12)$ & $4(12)$ \\
\hline $\mathrm{PPH}$ & $2(12)$ & $3(9)$ \\
\hline Other ${ }^{\#}$ & $1(6)$ & $4(12)$ \\
\hline \multicolumn{3}{|l|}{ Immunosuppresssion" } \\
\hline Cyclosporine & $16(94)$ & $29(88)$ \\
\hline Tacrolimus & $1(6)$ & $4(12)$ \\
\hline Azathioprine & $12(71)$ & $27(81)$ \\
\hline Mycophenylate & $3(18)$ & $5(15)$ \\
\hline Time post-transplant $^{+}$ & $1335(61-3104)$ & $786(19-2651)$ \\
\hline Previous BO or BOS & $2(12)$ & $5(15)$ \\
\hline Single lung transplant & $12(71)$ & $24(73)$ \\
\hline Double lung transplant & $4(24)$ & $8(24)$ \\
\hline Heart-lung transplant & $1(4)$ & $1(3)$ \\
\hline
\end{tabular}

Data are presented as $\mathrm{n}$, mean $\pm \mathrm{SD}, \mathrm{n}$ (\%) or median (range). M: male; $\mathrm{F}$ : female; IPF: idiopathic pulmonary fibrosis; COPD: chronic obstructive pulmonary disease; AAT: $\alpha_{1}$-antitrypsin deficiency; CF: cystic fibrosis; PPH: primary pulmonary hypertension; BO: bronchiolitis obliterans; BOS: bronchiolitis obliterans syndrome. \#: sarcoidosis, lymphangioleiomyomatosis, Eisenmenger's syndrome and post-adult respiratory distress syndrome fibrosis; ': one patient in both groups was enrolled in a trial comparing azathioprine and everolimus, all patients were receiving prednisone; ${ }^{+}: p=0.04$ (Mann-Whitney $\mathrm{U}$-test), all other comparisons between infected and uninfected patients were not significant.

vaccination, FLU serological results were excluded in patients who had received their FLU vaccination $<28$ days before the baseline serum draw. This led to the exclusion of 12 out of the 50 patients in the serological analysis of FLU.

\section{Statistics}

Categorical variables were analysed by Chi-squared test or Fisher's exact test. Medians were analysed with the MannWhitney U-test. Means were analysed with unpaired t-tests. A p-value of $<0.05$ was considered significant.

\section{RESULTS}

Tables 1 and 2 provide information on demographics and virus detection in the 50 study patients. As seen in table 1, the infected and uninfected groups did not differ in age, sex, underlying lung disease, type of transplant, type of immunosuppression, or in prior diagnoses of $\mathrm{BO}$ or BOS. However, patients with documented infection had a longer interval to transplant surgery (median 1,335 versus 786 days; $p=0.04$, Mann-Whitney U-test). Of the 50 study patients, 32 (64\%) had NW or BAL studied for respiratory viruses, including $44 \mathrm{NW}$ and five samples of BALF. Overall, laboratory evidence of respiratory viral infection was found in $17(34 \%)$ of the 50

\begin{tabular}{|c|c|c|c|c|c|}
\hline TABLE 2 & $\begin{array}{l}\text { Type of } \\
\text { patients }\end{array}$ & $\begin{array}{l}\text { irus, me } \\
\text { with infe }\end{array}$ & $\begin{array}{l}d \text { of } d \\
n\end{array}$ & ction ano & total \\
\hline \multirow[t]{2}{*}{ Virus } & \multicolumn{4}{|c|}{ Method of detection } & \multirow{2}{*}{$\begin{array}{l}\text { Infected } \\
\text { patients }\end{array}$} \\
\hline & Antigen & Culture & PCR & Serology & \\
\hline RSV & 1 & 1 & 5 & 7 & $8(16)$ \\
\hline Adenovirus & NA & 0 & NA & NA & $0(0)$ \\
\hline FLU & 2 & 3 & 4 & 8 & $10(20)$ \\
\hline Parainfluenza & NA & 1 & 1 & NA & $1(2)$ \\
\hline Total & & & & & $17(34)$ \\
\hline
\end{tabular}

patients during the study interval. Table 2 reveals the type of virus, the method of detection and the total number of patients infected with each virus. Infections with RSV and FLU were common, occurring in 16 and $20 \%$ of the study population, respectively. Two patients had multiple infections, one patient had concomitant FLU A and RSV infections and one patient had separate FLU A and RSV infections occurring $\sim 2$ months apart. PIV infection was only found in one patient; however, there was no antigen or serological testing available for this virus so this may be an underestimation of its true frequency. Adenovirus was not isolated in culture from any patient in this study. Antigen and serological testing was also not available for adenovirus. As can be seen in table 2, PCR and serology were the most sensitive techniques for detecting infection. In the case of RSV, there was considerable concurrence between PCR and serology. Four out of five patients with positive PCR had documented seroconversions. By contrast, only two of four patients with positive PCR for FLU A seroconverted to influenza A. Six patients had seroconversions to influenza A or B without having a positive PCR or positive cultures. In four out of these six patients, however, no NW samples were collected because the patients never reported symptoms during the study period. The other two patients were evaluated for respiratory symptoms, but recorded negative results on the virological tests.

Out of the five patients with RSV detected by PCR, three had type B and two had type A. The serology for RSV did not distinguish between RSV serotypes A and B. All four patients who were PCR positive for FLU had FLU A and three of these four infections were also confirmed by culture. However, the six seroconversions included four patients with FLU B infection, one of whom also seroconverted to FLU A. Out of the three patients who seroconverted to FLU A, two had serological rises to H1N1 Sydney, and one to H3N2 Beijing. The single PIV that was detected by PCR and isolated in culture was PIV 1.

Table 3 displays data on patients who had significant complications associated with viral respiratory infection. LRTI occurred in four $(24 \%)$ of the infected patients and led to hospitalisation in two (12\%). In all four cases, virus was detected in NW by culture and PCR in three patients and by 


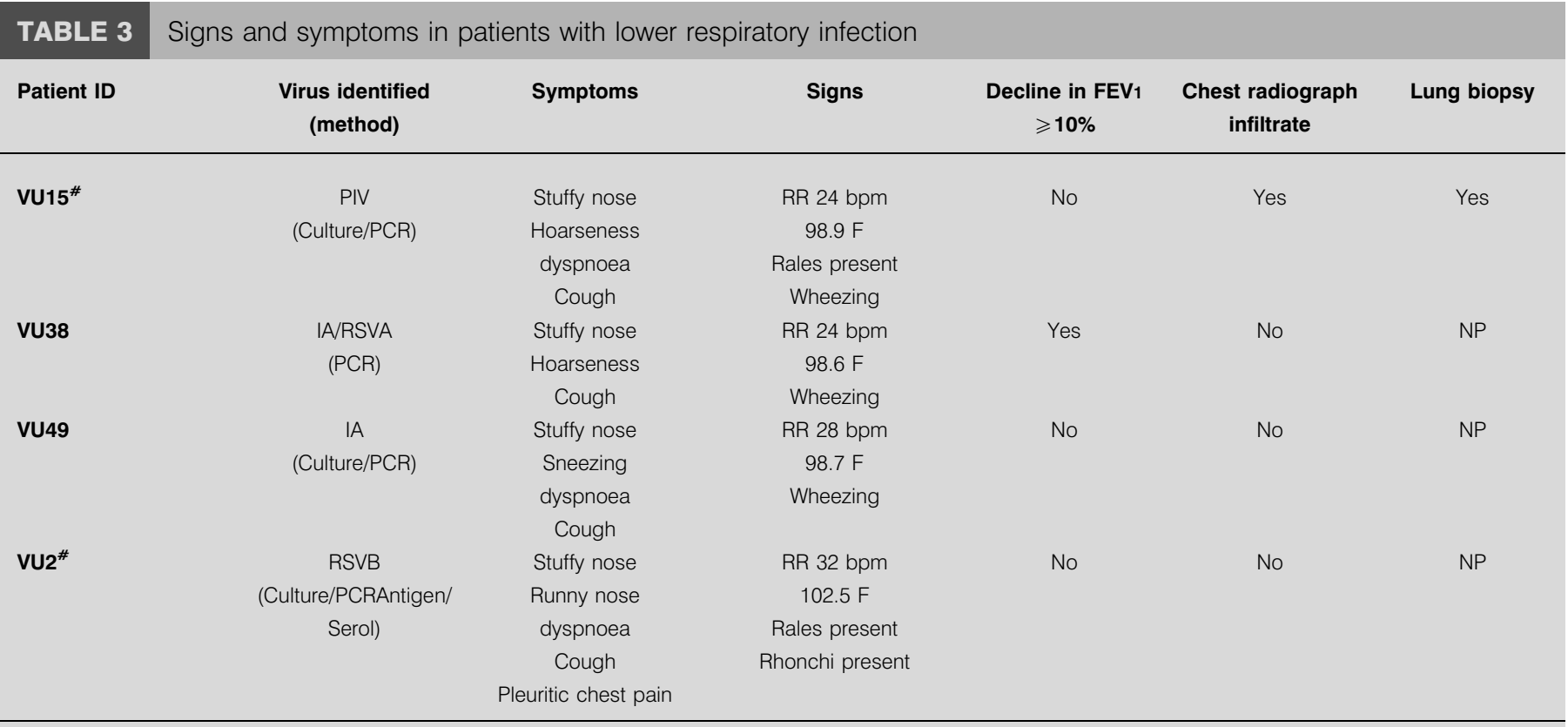

ID: identification; FEV1: forced expiratory volume in one second; PIV: parainfluenza virus; RR: respiratory rate breaths min $^{-1}$; bpm: beats per minute; $F$ : fahrenheit; IA:

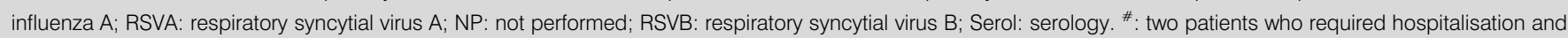
were treated with inhaled ribavirin.

PCR alone in one patient. One $(6 \%)$ of the 17 patients had pneumonia. This patient (VU15; table 3) underwent bronchoscopy with transbronchial biopsies and BAL 1 week after the NW culture turned positive for PIV. The biopsy showed pneumonia and PIV was detected from BAL by PCR, but not by culture. There were no deaths from respiratory viral infection in this population. Both patients who were admitted to the hospital were treated with aerosolised ribavirin.

Table 4 presents the evaluation of outcomes in the year following the end of the study. There were three deaths in the study population, two occurred in patients who had respiratory viral infection during the study. One of the patients died 6 months after the end of the study from an intraperitoneal haemorrhage and the other patient after $1 \mathrm{yr}$ from aspiration pneumonia following a cardiac procedure. The one death that occurred in a patient without documented respiratory viral infection was related to progressive pulmonary compromise in the setting of advanced $\mathrm{BO}$. The rates of

TABLE 4 Clinical outcomes $1 \mathrm{yr}$ after the end of the study

\begin{tabular}{lcc}
\hline Outcome & Infected $^{\#}$ & Uninfected $^{\#}$ \\
\hline Subjects & 17 & 33 \\
Death & $2(12)$ & $1(3)$ \\
Acute rejection & $0(0)$ & $5(15)$ \\
BO or BOS & $1(7)$ & $3(11)$ \\
\hline
\end{tabular}

Data are presented as $\mathrm{n}$ or $\mathrm{n}(\%) .{ }^{\top}:$ the denominators for this comparison are the patients who did not have prior BO or BOS (infected=15; uninfected=28).

\#: all comparisons $p=$ nonsignificant. acute rejection or new diagnoses of $\mathrm{BO}$ or BOS in the year after the study were not different between patients with and without respiratory viral infection. However, the one case of BO that occurred in a patient with respiratory viral infection was in a patient who had evidence of LRTI (VU49; table 3). Figure 1 shows selected virological data from the Vanderbilt University Hospital virology laboratory for five consecutive respiratory viral seasons beginning in 1997 and ending in 2002. Figure 1a represents the number and proportion of positive FLU cultures. Similar data for RSV antigens are shown in figure $1 \mathrm{~b}$. In both parts of the figure, data from the study year (1999-2000) is shown. As can be seen from this figure, the number of diagnoses of RSV and FLU virus infection in the study season was not unusually high or low compared with the surrounding years.

During the 5-month study interval two lung transplant recipients who were not eligible for the study, because they had been recently transplanted and were not yet discharged from the hospital, died of respiratory viral infection. One patient was diagnosed with RSV pneumonia a few days after transplantation. In retrospect, the patient had had mild coryza when admitted for the operation. It is suspected that the patient had upper airway infection with RSV before transplantation and the infection spread to the lung allograft shortly after transplantation. The other patient had primary graft failure and developed a urinary infection secondary to adenovirus 9 days after transplantation, which subsequently spread to the transplanted lung. The source of infection was not determined.

\section{DISCUSSION}

In the decade following the first heart-lung transplantation in 1981, surveys of infectious complications only made occasional 

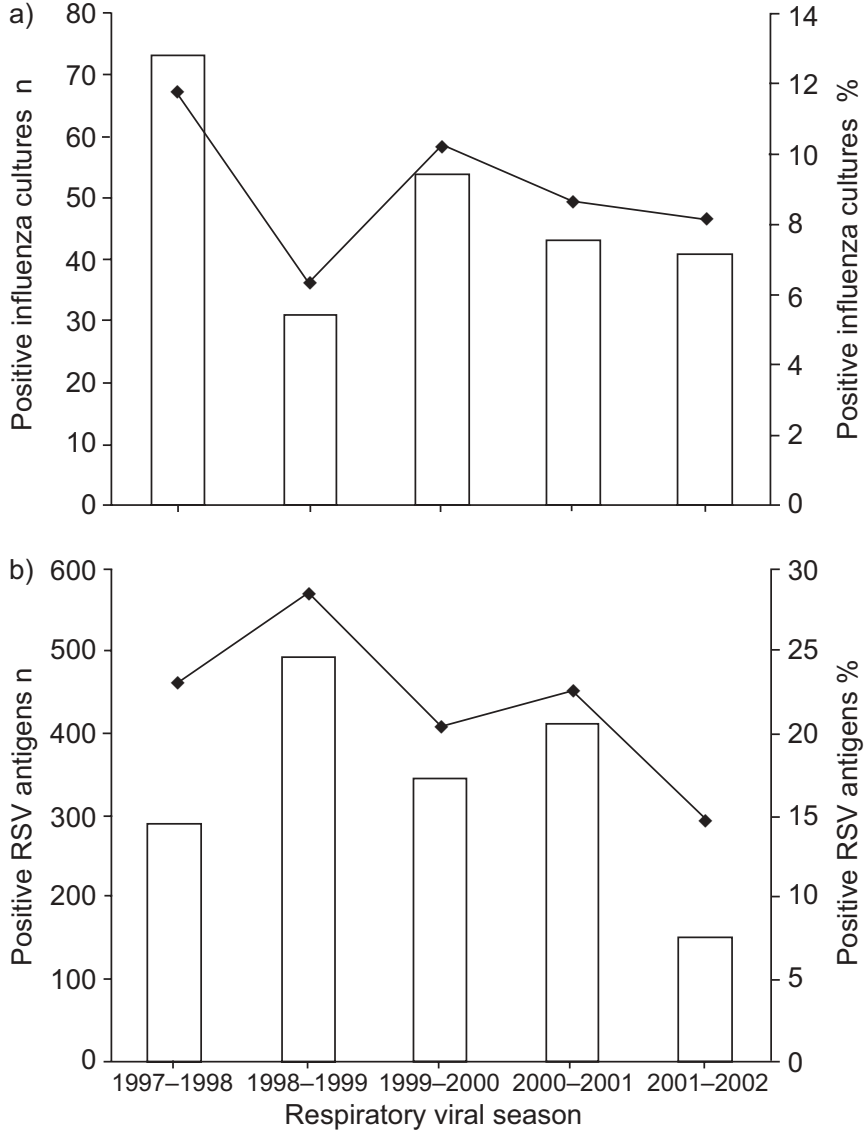

FIGURE 1. a) Number of positive influenza cultures $(\square)$ and the proportion of influenza positive cultures (-) in the Vanderbilt Hospital (Nashville, TN, USA) virology laboratory for five consecutive respiratory viral seasons. b) Number of positive respiratory syncytial virus (RSV) antigens ( $\square$ ) and the proportion of positive RSV antigens (-) in the Vanderbilt Hospital virology laboratory for five consecutive respiratory viral seasons.

note of respiratory viral infections. Since the 1990s, studies focusing on respiratory viral infections have shown that they are a cause of significant morbidity and mortality in lung transplant recipients [3-5]. These studies have accurately defined the clinical presentation and more serious outcomes of respiratory viral infections in this population, but have some limitations due to their retrospective design. Also, most studies did not employ more recently available diagnostic techniques, such as PCR, to determine the presence of viral infection. Recently, GARBINO et al. [15] studied lung recipients with a broad panel of PCR tests for respiratory viruses. However, only patients who had undergone bronchoscopy with available respiratory viral cultures were studied. Thus, it was not possible to calculate the incidence of respiratory viral infections in that study population or compare infected with uninfected patients. Respiratory viruses were detected by PCR in 55\% of 31 lung recipients who were thought to have respiratory viral infection by clinical criteria. Of the detected viruses, 53\% were rhinoviruses and the remainder were paramyxoviruses, orthomyxoviruses or adenoviruses.

The current study detected respiratory viral infection in onethird of clinically stable ambulatory lung transplant recipients who were followed intensively using multiple diagnostic modalities over a 5-month respiratory virus season. Although many of the 17 infected patients had only mild infections limited to the upper respiratory tract, the rate of lower respiratory tract involvement was $8 \%$ (four out of 50 ) over the 5-month study interval. This rate is somewhat higher than previously reported rates. For instance, the average yearly rate of lower respiratory paramyxoviral infection reported by WENDT et al. [4] in a 7-yr retrospective evaluation was 3\%. Similarly, PALMER et al. [3] reported an average annual incidence rate of $1.6 \%$ for the occurrence of RSV, PIV and adenovirus infection in a 5-yr interval. The present findings do not merely reflect an unusually high rate of respiratory viral infection during the 1999-2000 season, as is shown by the 5 yrs of data from the Vanderbilt virology laboratory (fig. 1). Indeed, the rate of lower respiratory viral infection may be an underestimation of the actual yearly rate in the population, because the current study was only conducted during the 5month respiratory season from November through to March and the diagnostic efforts were on the viruses which have been known to cause severe disease in lung recipients, notably RSV, PIV, FLU and adenovirus. The present authors specifically did not look for rhinoviruses or coronviruses. Also, metapneumovirus was not investigated, as it had not been described at the time of the study. In recent studies these viruses have emerged as probable respiratory pathogens in lung transplant recipients and should be included in future studies [15, 20-22]. Future studies might also include techniques to detect atypical pathogens, such as Chlamydia pneumonia and Mycoplasma pneumonia, as infection with these pathogens may mimic respiratory viral infection [15].

In the current study, the most common viral infection, FLU, was also associated with the least morbidity. Other studies have shown similar results, including recent work by VILCHEZ et al. [23] who evaluated 15 lung transplant recipients infected and hospitalised with FLU. None of the FLU-infected patients in that study developed respiratory failure despite the fact that five out of 15 (33\%) had clinical pneumonia. GARANTZIOTIS et al. [24] described FLU pneumonia in three lung transplant recipients. All three patients had significant radiographical changes consistent with pneumonia and all recovered from their acute viral illness but developed late chronic rejection. In the current study two patients had lower respiratory involvement but neither had pneumonia or required hospitalisation. One of these patients (patient 49) was diagnosed with BOS $<1 \mathrm{yr}$ after infection. As the patient was not hospitalised and did not undergo bronchoscopy the case might have been missed in a retrospective study.

A plausible explanation for the low acute FLU morbidity in the current study is the high immunisation rate in the transplant population. The current authors attempt to vaccinate all lung transplant recipients at the Vanderbilt University Medical Center on a yearly basis. The authors were able to document FLU vaccination in $\geqslant 80 \%$ of the 50 study patients. A prospective study would be necessary to see whether consistent yearly FLU vaccination truly reduces infection rates or attenuates morbidity in lung recipients. Such a study would require monitoring of a large number of patients for the adequacy of serological responses; the patients would also need to be followed over a number of years. 
Although the overall rates of respiratory viral infection in the current study were higher than in previous lung transplant reports, the rates of severe LRTIs were similar to those studies, as evidenced by the frequency of pneumonia $(2 \%)$ and hospitalisation $(4 \%)$. No deaths from respiratory viral infection were seen in the ambulatory study patients, most of whom were healthy. However, two deaths were diagnosed due to respiratory viral infection in patients who had recently received transplants and were not eligible for entry into the prospective study. It is likely that patients are more vulnerable during this early post-transplant interval because they receive higher doses of immunosuppression, have lower pulmonary reserve and may have ischaemic injury to the transplanted lung. POHL et al. [25] noted that RSV infection in children with liver transplants was more likely to be fatal if it occurred early after transplantation.

Several authors have associated the occurrence of respiratory viral infection with subsequent development of $\mathrm{BO}$ or $\mathrm{BOS}$ [11]. In the study by BILLINGS et al. [11], the occurrence of BO or BOS was specifically linked with preceding viral LRTI. The current study did not show any association between respiratory viral infection and BO or BOS, but most of the cases had infection limited to the upper respiratory tract and there were only four cases of LRTI.

It is unclear why respiratory viral infections in the present study were more common in patients who were later posttransplantation, though it did not appear to be due to a higher rate of $\mathrm{BO}$ or $\mathrm{BOS}$ in this group. One possible explanation is that the patients who had been transplanted more recently may have been more likely to attend the centre for minor symptoms. They may have been more likely to be infected by less virulent organisms, such as rhinoviruses, as opposed to the more virulent orthomyxoviruses and paramyxoviruses. A larger and more comprehensive prospective study, which encompasses more viruses, should be able to evaluate this possibility.

In conclusion, the current study represents one of the few prospective studies that have used multiple diagnostic modalities, including PCR, to evaluate the incidence and impact of respiratory viral infections in lung transplant recipients. It shows that studies that only look at clinically diagnosed patients may underestimate the underlying rate of respiratory tract infection. While this result is not surprising in itself, it does illustrate that a complete picture of the morbidity and immunological consequences of respiratory viral infection in lung transplantation will only be achieved with prospective population-based studies. These studies would ideally use both conventional and molecular techniques to detect a large number of different respiratory viruses at multiple centres over many years.

\section{ACKNOWLEDGEMENTS}

The current authors would like to thank T. Johnson who performed the respiratory syncytial virus serologies in the laboratory of B. Graham. The influenza serologies were carried out by E. Sannella in the laboratory of J. Crowe. S. Bozeman assisted in patient monitoring and the Vanderbilt University Hospital virology laboratory performed the cultures and antigen studies.

\section{REFERENCES}

1 Kramer MR, Marshall SE, Starnes VA, Gamberg P, Amitai Z, Theodore J. Infectious complications in heartlung recipients. Arch Int Med 1993; 153: 2010-2016.

2 Dauber JH, Paradis IL, Dummer JS. Infectious complications in pulmonary allograft recipients. Clin Chest Med 1990; 11: 291-308.

3 Palmer SM, Henshaw NG, Howell DN, Miller SE, Davis RD, Tapson VF. Community respiratory viral infection in adult lung transplant recipients. Chest 1998; 113: 944-950.

4 Wendt CH, Fox JMK, Hertz MI. Paramyxovirus infection in lung transplant recipients. J Heart Lung Transplant 1995; 14: 479-485.

5 Holt ND, Gould FK, Taylor CE, et al. Incidence and significance of noncytomegalovirus viral respiratory infection after adult lung transplantation. J Heart Lung Transplant 1997; 16: 416-419.

6 Ohori NP, Michaels MG, Jaffe R, Williams P, Yousem SA. Adenovirus pneumonia in lung transplant recipients. Human Pathology 1995; 26: 1073-1079.

7 Matar LD, McAdams HP, Palmer SM, et al. Respiratory viral infections in lung transplant recipients: radiological findings with clinical correlation. Radiology 1999; 213: 735-742.

8 Bridges ND, Spray TL, Colins MH, Bowles NE, Towbin JA. Adenovirus infection in the lung results in graft failure after transplantation. J Thorac Cardiovasc Surg 1998; 116: 617-623.

9 Winter JB, Glouw AS, Groen M, Widlevuur C, Prop J. Respiratory viral infections aggravate airway damage caused by chronic rejection in the rat lung. Transplantation 1994; 57: 418-422.

10 Hohlfeld J, Niedermeyer J, Hamm H, Schafers HJ, Wagner TO, Fabel H. Seasonal onset of bronchiolitis obliterans syndrome in lung transplant recipients. J Heart Lung Transplant 1996; 15: 888-894.

11 Billings JL, Hertz MI, Savik K, Wendt CH. Respiratory viruses and chronic rejection in lung transplant recipients. J Heart Lung Transplant 2002; 21: 559-566.

12 Falsey AR, Cunningham CK, Barker WH, Kouides RW, Yuen JB, Menegus M. Respiratory syncytial virus and influenza A infections in the hospitalized elderly. J Infect Dis 1995; 172: 389-394.

13 Fan J, Henrickson KJ, Savatski LL. Rapid simultaneous diagnosis of infections with respiratory syncytial viruses $A$ and $\mathrm{B}$, influenza viruses $\mathrm{A}$ and $\mathrm{B}$, and human parainfluenzaviruses types 1,2 and 3 by multiplex quantitative reverse transcription-polymerase chain reaction-enzyme hybridization assay (hexaplex). Clin Inf Dis 1998; 26: 1397-1402.

14 Falsey AR, Formica MA, Walsh EE. Diagnosis of respiratory syncytial virus infection: comparison of reverse transcription-PCR to viral culture and serology in adults with respiratory illness. J Clin Microbiol 2002; 40: 817-820.

15 Garbino J, Gerbase MW, Wunderli W, et al. Lowerrespiratory viral illnesses: improved diagnosis by molecular methods and clinical impact. Am J Respir Crit Care Med 2004; 170: 1197-1203.

16 Brumble LM, Milstone AP, Loyd JE, et al. Prevention of cytomegalovirus disease after lung transplantation: results 
using a unique regimen employing delayed ganciclovir. Chest 2002; 121: 407-414.

17 Yousem SA, Berry GJ, Cagle PT, et al. Revision of the 1990 working formulation for the classification of pulmonary allograft rejection: Lung Rejection Study Group. J Heart Transplant 1996; 15: 1-15.

18 Johnson TR, Johnson JE, Roberts SR, Wertz GW, Parker RA, Graham BS. Priming with secreted glycoprotein G of respiratory syncytial virus (RSV) augments interleukin-5 production and tissue eosinophilia after RSV challenge. $J$ Virol 1998; 72: 2871-2880.

19 Snyder M, Banks S, Murphy BR. Determination of antibody responses to influenza virus surface glycoproteins by kinetic enzyme-linked immunosorbent assay. J Clin Micro 1988; 26: 2034-2040.

20 Kumar D, Erdman D, Keshavjee S, et al. Clinical impact of community-acquired respiratory viruses on bronchiolitis obliterans after lung transplant. Am J Transplant 2005; 5: 2031-2036.
21 Larcher C, Geltner C, Fischer H. Human metapneumovirus infection in lung transplant recipients: clinical presentation and epidemiology. J Heart Lung Transplant 2005; 24: 1891-1901.

22 Sumino K, Agapov E, Pierce R. Detection of severe human metapneumovirus infection by real-time polymerase chain reaction and histopathological assessment. J Infect Dis 2005; 192: 1052-1060.

23 Vilchez R, McCurry K, Dauber J, et al. Influenza and parainfluenza respiratory viral infection requiring admission in adult lung transplant recipients. Transplantation 2002; 73: 1075-1078.

24 Garantziotis S, Howell DN, McAdams HP, Davis RD, Henshaw NG, Palmer SM. Influenza pneumonia in lung transplant recipients: clinical features and association with bronchiolitis obliterans syndrome. Chest 2001;119: 1277-1280.

25 Pohl C, Green M, Wald ER, Ledesma-Medina J. Respiratory syncytial virus infections in pediatric liver transplant recipients. J Inf Dis 1992; 165: 166-169. 